\title{
Reflex inhibition and reflex strength
}

\author{
JAMES R. ISON and LEVI A. REITER \\ University of Rochester, Rochester, New York 14627
}

\begin{abstract}
In Experiment 1, the amplitude of the acoustic startle reflex in rats was shown to increase linearly with increases in the intensity of the eliciting stimulus (Se). A preliminary stimulus (Si), a light flash, inhibited the reflex. The amplitude of the reflex was reduced by an equal amount regardless of Se intensity. In Experiment 2, the amplitude of the same reflex was shown to have a biphasic relation to the intensity of a white noise background $(\mathrm{Sn})$ and reflex amplitudes peaked at the intermediate noise levels. Although the vigor of the reflex elicited in the presence of the most intense noise approximated that elicited by the weak Se in Experiment 1, in this condition the reflex was unaffected by the occurrence of the otherwise inhibitory light flash. It has been previously hypothesized that the decrement in reflex vigor obtained at high $\mathrm{Sn}$ results because sensory masking of Se by Sn reduces the effective intensity of Se. The different effect of Si in these two conditions suggests that this hypothesis is incorrect. A hypothetical neural model was devised to account for the difference between the two reflexes in their susceptibility to a preliminary stimulus, and the model was extended to other effects of stimulus strength and background noise on reflexive behavior.
\end{abstract}

The strength of the acoustic startle reflex in the rat is substantially affected by the intensity of the eliciting stimulus (Se), by the level of the background noise present at the time of reflex elicitation (Sn), and by the occurrence of irrelevant stimuli just prior to that time (Si). Louder eliciting stimuli elicit more vigorous startle reactions (Hoffman \& Wible, 1970); startle reactions are most pronounced at intermediate background noise intensities, being diminished in amplitude at both high and low extremes (Davis, 1974; Ison \& Hammond, 1971); and irrelevant stimuli presented at appropriate lead times before Se reduce the amplitude of the reflex (Hoffman \& Searle, 1965; Ison \& Hammond, 1971; and many subsequent reports).

Stitt, Hoffman, and Marsh (1976) examined the effectiveness of a fixed Si on reflexes of different strengths, these differences resulting from variation in the intensities of Se. They found that Si diminished the amplitude of the reflex by a constant amount regardless of the intensity of Se. Hoffman and Ison (1980) describe a simple linear subtractive model of reflex inhibition to account for this finding. It is first assumed that the amplitude of a motor response reflects the number of motor units activated by Se, that number being a monotonic increasing function of $\mathrm{Se}$ intensity. It is then assumed that a given $\mathrm{Si}$ inactivates the same units regardless of the intensity of Se and regardless of the number of units associated with the presence of any particular Se. These two

This work was supported by Research Grants NS-12443 and ES-01248 from the National Institutes of Health. Reprints may be obtained from Professor Ison, Psychology Building. University of Rochester. Rochester. New York 14627. simple assumptions produce an invariant decrement in reflex amplitudes regardless of reflex strength, unless the amount of inhibition produced by $\mathrm{Si}$ is large relative to the strength of the reflex (i.e., a "floor effect" may deform the relationship).

The present experiment assessed the generality of this invariance by examining the effectiveness of $\mathrm{Si}$ on the variously sized reflexes that occur in different noise backgrounds. The descending upper limb of the biphasic curve relating response amplitude to $\mathrm{Sn}$ intensity has been ascribed to a process akin to that responsible for the relationship between Se intensity and reflex strength. It has been proposed that, at noise levels higher than the optimum, the background noise increasingly masks Se and reduces its effective intensity (Davis, 1974; Ison \& Hammond, 1971): an increment in Sn is formally identified with a decrement in Se. If this hypothesis is correct, then, in this range of relatively high noise levels past the optimal value, the preliminary stimulus should subtract a constant amount from reflex amplitude.

\section{METHOD}

\begin{abstract}
Subjects
The subjects were albino male rats of the Holtzman strain weighing $350-500 \mathrm{~g}$ at the time of testing. They were maintained on an ad-lib food and water schedule.

Apparatus

The rats were restrained during testing in a small perforated Plexiglas stabilimeter cage $(8 \times 6 \times 9 \mathrm{~cm}$ high $)$ that was mounted on compression springs in an aluminum frame. An accelerator mounted on the back of the stabilimeter detected the force exerted by the rat on the cage floor. The output of the accelerometer was amplified on a Beckman dynograph and displayed on a Tektronix $5103 \mathrm{~N}$ storage oscilloscope. The reaction was recorded as millimeters of beam deflection from baseline, peak to peak.
\end{abstract}


Housed with the stabilimeter within a large double-walled IAC room (approximately $3 \times 4 \mathrm{~m}$ ) were two speakers, one to provide a continuous background white noise (Sn), the other to produce the acoustic startle stimulation (Se). The frequency response of the white noise was within $2 \mathrm{~dB}$ of being flat from .1 to $10 \mathrm{kHz}$. Four 24-V dc light bulbs, one mounted near each corner of the cage, provided a dimly illuminated background and a visual preliminary stimulus (Si). All control and recording equipment were kept outside the IAC room. Acoustic stimuli were delivered through electronic switches, and stimulus presentation was controlled by solid state electronic timers. (Details of the apparatus are given in Russo, Reiter, \& Ison, 1975.) The startle stimuli were $10-\mathrm{kHz}$ tones, ranging from 95 to $125 \mathrm{~dB}$ in intensity (all sound levels are re $20 \mu \mathrm{N} / \mathrm{m}^{2}$ ). They were $25 \mathrm{msec}$ in duration, with a rise-decay time of $5 \mathrm{msec}$. The visual prestimulus was a 40-fc light flash, $25 \mathrm{msec}$ in duration. Earlier work (Ison \& Hammond, 1971) has established that the effective component of this stimulus is the change in intensity of the light. The room was otherwise dimly illuminated by a $60-\mathrm{W}$ red light bulb, which was sufficient light for the TV system used to monitor the subject's behavior. Trials were given about $20 \mathrm{sec}$ apart, unless the animal was moving or in an awkward position at the designated time.

\section{Procedure}

In the first experiment, the rat was allowed 5 min adaptation to apparatus and background noise, given two startle stimuli, and then run through the experimental series. All conditions were run within subjects. Three intensities of a startle stimulus were given: 95,105 , and $125 \mathrm{~dB}$. These were presented either alone, constituting a control trial (Se-alone), or following a preliminary light flash that had a lead time of $60 \mathrm{msec}(\mathrm{Si}-\mathrm{Se})$. There were, thus, six different types of trials. Each type was given six times, with the order of presentation determined by a 6 by 6 Latin square, different for each subject. A 70-dB background noise was continuously present.

In the second experiment, the startle stimulus was a $110-\mathrm{dB}$ tone. The visual prestimulus was the $40-\mathrm{fc}$ light flash. Four intensities of background noise were used: $45,60,75$, and $90 \mathrm{~dB}$. The rat was allowed $5 \mathrm{~min}$ adaptation to the apparatus with no background noise present (an ambient noise level of $27 \mathrm{~dB}$ ) followed by two startle trials. After this, the experiment proper began. All conditions were run within subjects. A particular background stimulus was turned on for $30-40 \mathrm{sec}$, and startle stimuli were given approximately in the middle and then just before the end of the noise. In one location, the startle stimulus was given alone, and in the other, it was preceded by the light flash with an intervening interval of $60 \mathrm{msec}$. The order of these two conditions was varied randomly, with the restriction that they occur equally often at each location for each background level. Following the second startle stimulus, the background was turned off and the next one imposed after a rest period of approximately $5 \mathrm{sec}$. The order of background conditions was taken from 4 by 4 Latin squares. Each background condition occurred eight times.

\section{RESULTS}

The left panel in Figure 1 shows the mean startle amplitudes at each of the three Se intensities. As anticipated, reflex strength was affected by this variable: it increased in approximately linear progression with linear changes in intensity. The presence of the light flash had a major depressive influence in the reflex at each intensity. At the highest intensity, the 10 -mm decrement in mean response amplitude produced by $\mathrm{Si}$ approximated the size of the reflex elicited by the $105-\mathrm{dB}$ stimulus and was greater than that

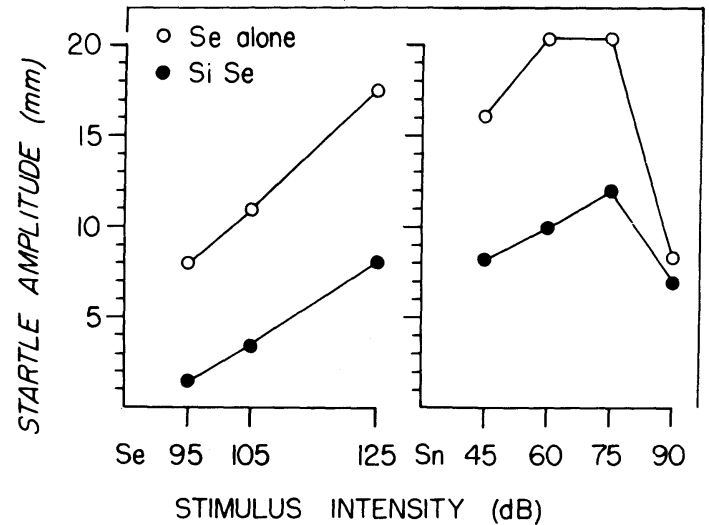

Figure 1. Mean startle amplitudes to the eliciting tone (Se) and to the tone preceded by a light flash (SiSe). In the left panel, Se intensity was varied (Experiment 1). In the right panel, background noise level (Sn) was varied (Experiment 2).

elicited by the 95-dB stimulus. This "floor effect" necessarily prevented an exact constant subtractive consequence of the initial stimulus (Hoffman \& Ison, 1980), although statistical analysis did not reveal any significant deviation from the ideal (for the interaction of Intensity by Light Flash, $F<1$ ). The overall effect of stimulus intensity was significant $[\mathrm{F}(2,10)=15.29$, $p<.01$ ], as was the decremental effect of the light flash $[\mathrm{F}(1,5)=152.88, \mathrm{p}<.01]$.

The major service provided by this initial experiment was to establish baseline expectations for the effectiveness of the light flash with different eliciting stimuli. These provided a benchmark against which the functions obtained from varying background intensity could be compared. The data obtained from varying Sn intensity are given in the right-hand panel of Figure 1. The biphasic relationship between reflex strength on Se-alone trials and Sn intensity was the same as that obtained in prior work, with a peak in the region of 60-75 dB. A similar, but less pronounced, biphasic function occured on the trials on which Se was preceded by the light flash. Analysis of variance of these data revealed that the effect of background noise was reliable $[\mathrm{F}(2,27)=8.97, \mathrm{p}<.01]$, as was the inhibitory effect of the light $[\mathrm{F}(1,9)=17.81, \mathrm{p}<.01]$. In this case, however, the effectiveness of the light clearly depended on the intensity of the background noise, whereas in the earlier analysis its effect was independent of the intensity of the eliciting stimulus. The interaction of the effect of the light flash and the effect of noise intensity was reliable $[F(3,27)=$ $7.11, \mathrm{p}<.01]$. The linear and quadratic trends present in the background effect $(p<.05)$ both interacted reliably with the occurrence of the light $(p<.05)$.

In the absence of the light flash, the startle reflex amplitudes obtained with the 45-, 60-, and 75-dB backgrounds, at means ranging from about 17 to $20 \mathrm{~mm}$, were about equivalent to the mean for the 
125-dB stimulus condition of the first experiment. The depressive effect of the light flash was about the same in each of these conditions. At the $90-\mathrm{dB}$ background level, the size of the response, at about $8 \mathrm{~mm}$, was reduced to a value that approximated that seen earlier for the 95-dB eliciting stimulus. In the first experiment, the light was so effective that the small response to the 95-dB stimulus was about completely inhibited. In contrast, in the second experiment the light was almost without effect, and the responses in the presence of the $90-\mathrm{dB}$ background noise were about the same in the Se-alone control condition as they were in the Si-Se paired trial.

\section{DISCUSSION}

The inhibitory effect of a visual stimulus, the biphasic effect of background noise, and the increase in reflex vigor with an increase in the strength of the eliciting stimulus have all been shown in prior experiments. The new finding is that whereas inhibition is not influenced by changes in the intensity of the eliciting stimulus, it is very much dependent on the level of the background noise. When the acoustic startle reflex is weak because of the presence of an intense noise background, then it is not further depressed by a preliminary stimulus. In contrast, when the acoustic startle reflex is weak because a weak eliciting stimulus is used, then that same preliminary stimulus is very effective in depressing the response. This difference in susceptibility of these two "phenotypically" similar reflexes to preliminary stimuli must have significance for our understanding of the terminal decrement in the acoustic startle reflex produced by intense background noise. In particular, it must be that the earlier masking hypothesis (Davis, 1974; Ison \& Hammond, 1971) is inappropriate. If the two reflexes were of the same strength because the high background noise works through a process functionally equivalent to that engendered by a weak eliciting stimulus, then the reflexes should be similarly affected by initial stimuli. That they are not reveals the inadequacy of this hypothesis and suggests, instead, that the apparently similar responses are produced by different underlying mechanisms.

The sensory masking hypothesis is not readily reconciled with other sorts of findings, besides those obtained here. Perhaps most telling is the fact that judgments of signal loudness are primarily affected by masking noises which equal or exceed the intensity of the signal. Masking is absent when the signal exceeds the mask by as little as 25-30 dB (Steinberg $\&$ Gardner, 1937). The same graded effect can be shown for reflex inhibition (Reiter \& Ison, in press). In that experiment, we varied the intensity of initial stimulus, a tone, relative to a unilateral masking noise. The effectiveness of the masked tone in inhibiting a reflex was minimal when tone and mask were of the same intensity but normal when the tone exceeded the mask by about $25 \mathrm{~dB}$. So, for both psychophysical judgments of loudness and for reflex modulation, the occurrence of masking depends on intensities of signal and noise being within a range no greater than about $20 \mathrm{~dB}$. In contrast, for reflex elicitation the downturn of the biphasic function may occur when the noise is $50 \mathrm{~dB}$ less than the signal, suggesting that something other than sensory effectiveness is impaired.

Other experiments indicate that reflex facilitation and depression produced by noise vary with the strength of the reflex regardless of how reflex strength is manipulated. The interaction is not restricted to variation produced by differences in signal strength. Habituation (Cory \& Ison, 1979; Davis, 1974) and cortical ablation (Ison \& Silverstein, 1978), as well as variation in the intensity of the eliciting stimulus (Davis, 1974), all affect reflex vigor and all similarly influence the facilitatory effect of background noise level. The sensory masking hypothesis is seemingly appropriate for the effects of variation in the intensity of the eliciting stimulus (at least when stimulus and mask are of similar intensities), but there is little reason to suppose that a similar process underlies the effects of habituation and cortical ablation. (Although it has been hypothesized that habituation reduces the subjective intensity of the repeated stimulus, this notion was not supported by a direct test; Russo et al., 1975.)

For these several reasons, then, we believe that it is time to drop the sensory masking hypothesis as the general explanation of the downturn in the function relating acoustic startle behavior to the intensity of the background noise level. We realize that the masking process is increasingly appropriate as the noise level approaches and then equals the intensity of the eliciting signal. But, for a general account of the phenomenon, appropriate to those moderate noise conditions in which it is typically observed, a different account is necessary.

In the first description of the biphasic function (Ison \& Hammond, 1971), the initial ascending portion was attributed to "arousal." Two possibilities were put forward as candidates for the descending portion. One was the now familiar sensory masking hypothesis. The second was that reflex depression resulted from "overarousal" at intense noise levels. This second hypothesis has been neglected in the intervening years, but the failure of the alternative hypothesis suggests that it deserves some further consideration. A simple physiological mechanism underlying "arousal" can be reasonably applied to the ascending portion of the reflex function; its exaggeration, which then yields reflex depression, is also plausible and may provide a coherent account of the several reflex phenomena that follow from changes in background noise.

One process that may underlie differences in 
"arousal" is a change in the excitability of neurons involved in motor output. In the motor pathways, reflex facilitation derives from a partial depolarization or increased conductance of the neurons, so that subsequent input is more likely to be effective in driving the cell past its threshold for discharge. Facilitation of the rabbit's nictitating membrane (NM) reflex by auditory stimuli seems to result from this sort of process (Young, Cegavske, \& Thompson, 1976). The rat's acoustic startle reflex and the rabbit's cutaneous NM reflex respond similarly to antecedent noise conditions (Ison \& Leonard, 1971), and so it is not unreasonable to ascribe facilitation of the startle reflex to a similar mechanism which provides for what we call "arousal" at a molar level of analysis. Given this sort of underlying mechanism, the meaning of "overarousal" is unclear: It is that excitability has increased past the point at which the discharge threshold is exceeded just from the imposition of the noise alone. The motor units are activated before the eliciting stimulus is presented. We have reported that variation in background noise level does produce a systematic and correlated variation in general activity with levels of noise characteristic of reflex experiments (Ison \& Silverstein, 1978). This observation suggests that overarousal does occur, if we assume that the neural elements responsible for general activity in response to background noise are those involved in the acoustic startle reflex.

The only difference between noise-evoked activity and the startle reflex is that, in reflex elicitation, a brief synchronous burst of firing is responsible for the abrupt overt response, whereas prolonged noise produces a continual asynchronous firing of the neural elements, which yields general activity. The identity of these elements is important to our analysis because one consequence of cell discharge is a period of depressed reactivity. In some measure, intrinsic cellular events are responsible for this depression, but largely it results from collateral feedback connections working through inhibitory interneurons (see, e.g., Shepherd, 1979, pp. 139-140), which may affect both the neurons that initiate activity and their neighbors. Thus activation of the motor units by the noise makes them less likely to be discharged by the subsequent imposition of the startle stimulus.

The simple account of the biphasic function is this: At low noise levels, motor units are excited and more readily fire, given the eliciting stimulus. At high noise levels, motor units spontaneously discharge and then less readily fire, given the eliciting stimulus.

This explanation must be expanded with some further assumptions in order to explain the effects of the several variables that interact with background noise in determining reflex behavior.

The first interaction has to do with the intensity of the eliciting stimulus: The optimal noise value is greater with more intense stimuli (Davis, 1974). This finding is paradigmatic for the sensory masking hypothesis, whereby it is understood as a demonstration that a more intense signal is less affected by background noise. The finding, however, is equally explicable given the overarousal hypothesis, for here the refractory-like inhibition can be seen as the motor analog of masking. An additional assumption necessary for this explanation is that the reflex elements do not have equal sensitivities and are thus graded in their probabilities of discharge to noise and to eliciting stimuli. The most sensitive elements would show an early increase in excitability with an increase in background noise, but then would quickly become refractory. Less sensitive elements would have both processes delayed. Associated with these differences, the background noise function for weak eliciting stimuli, which excite only the most sensitive elements, would show an early peak in reflex strength. This peak would reflect only the excitability curve for the most sensitive elements. In contrast, the behavioral function for more intense stimuli would reflect the excitability curves for elements of various degrees of sensitivity: The early portion would show the effect of the increase in excitability of the most sensitive elements; a central portion would reflect a possible stable state characterizing the loss in excitability of more sensitive elements counterbalanced by the correlated increase in excitability of the less sensitive elements; and a terminal decline would reflect the declining excitability of the least sensitive elements at the highest levels of background noise.

The two other interactions involve habituation and the effects of cortical lesions: The optimal noise value for facilitation is reduced when the eliciting stimulus is habituated (Cory \& Ison, 1979; Davis, 1974) and increased in animals with cortical lesions (Ison \& Silverstein, 1978). The assumption that the reflex elements are differentially sensitive to acoustic stimuli can be used to explicate these interactions as well, if it is further assumed that the elements least sensitive to the excitatory effects of stimuli are the ones most affected by inhibitory effects of processes related to these variables. Take the case of the eliciting stimulus of moderate strength. Under normal conditions, the optimal noise value for this stimulus is relatively high because the decline in excitability of the most sensitive elements is counteracted by a correlated increase in excitability of less sensitive elements. If, however, these less sensitive elements have their excitability functions depressed by virtue of their history of repetitive discharge (i.e., because of habituation), then that compensating increase will not occur. Therefore, the optimal noise value must necessarily decline.

The argument for the effect of the cortical lesions is similar. These lesions result in greater reflex vigor, 
which is presumed to occur because the reflex elements are released from chronic inhibition (Hammond, 1974). We assume, then, that it is the less excitable elements which are chronically inhibited, just as it is these elements which are most susceptible to the inhibiting consequences of habituation. When inhibition is removed following brain damage, these released elements provide a further compensatory increase in excitability with the increase in background noise level. As a consequence, the optimal value must shift upwards compared with the normal value.

These several explanations of the background function and the variables with which it interacts do not deal, so far, with the experimental vehicle that led to their introduction. The overarousal hypothesis has not yet been applied to our present observation that the reflex that is depressed by its being elicited in the presence of prolonged intense noise cannot be further depressed by a preliminary flash of light. There is a question, then, whether the overarousal hypothesis can explain this finding, as well as whether the sensory masking hypothesis, or some variant thereof, might not also be able to account for the effect. Actually, masking could explain the data in one of two ways, one more interesting than the other. The less interesting explanation is that the finding is an artifact - that the light flash is effective only because of some associated acoustic cue which is then masked at the high noise intensity. Although some methods for delivering visual stimuli are contaminated by acoustic correlates, the present procedures are the same as those used in Ison and Hammond (1971). This earlier report conducted control procedures to show that visual rather than acoustic effects were responsible for the effects. The second explanation is to extend the masking model from an intradimensional to an extradimensional process, so that, for example, an intense noise degrades the visual system as well as the acoustic system. Again, there are two versions of this, one less interesting than the other. The less interesting is that, in the intense noise, the animal adopts a defensive posture which also prevents its reception of the light flash. We saw nothing like this during the experiment. The other version is that the intense noise activates a central inhibiting process which gates out incoming signals from the variety of modalities. This explanation is potentially very interesting and could account for the decline both in the effectiveness of the visual stimulus in inhibiting the reflex and in that of the acoustic stimulus in eliciting the reflex. We cannot dismiss this alternative account, but we should point out that it is considerably different from the original intradimensional masking hypothesis at which the present experiments were aimed.

The overarousal hypothesis is consistent with the present findings. The important difference between the phenotypically similar reflexes that appear in high noise levels, vs. weak eliciting stimuli, lies in the identities of the reflex elements that underlie the two responses and in their differential sensitivity to preliminary stimuli. The sample of reflex elements that are activated by a weak stimulus is composed of sensitive reflex elements. These elements are also sensitive to preliminary stimuli, which, therefore, are very effective in inhibiting this particular reflex. In contrast, the sample of reflex elements that underlies the response elicited in the presence of an intense background noise is composed of relatively insensitive reflex elements, the more sensitive elements having been discharged by the noise. The reflex elements that are relatively insensitive to the background noise are similarly relatively insensitive to the preliminary stimulus. Therefore, in this condition, the preliminary stimulus has little effect on the response.

Although rooted in the actual synaptic mechanisms, the analysis of the background function we propose is entirely molar and hypothetical. It is consistent with these molecular mechanisms, but, at present, its major interest derives from an attention to a variety of behavioral findings rather than any detailed correspondence with measures of activity in the physiological substrate. However, identification of the location of this substrate is progressing, as is indicated in the recent work of Davis and his associates (e.g., Gendelman \& Davis, 1979). The model presented here may provide a useful conceptualization for the physiological analysis of these behavioral effects and the further characterization of the neural mechanisms that produce them.

\section{REFERENCES}

Cory, R. N.. \& Ison, J. R. Persistent effect of noise on the acoustic startle reflex in the rat. Animal Learning \& Behavior, 1979, 7, 367-371.

Davis, M. Signal-to-noise ratio as a predictor of startle amplitude and habituation in the rat. Journal of Comparative and Physiological Psychology, 1974, 86, 312-325.

Gendelman, D. S., \& Davis, M. The primary acoustic startle circuit in the rat. Society for Neuroscience Abstracts, 1979. 5,494

Hammond. G. R. Frontal cortical lesions and prestimulus inhibition of the rat's acoustic startle reaction. Physiological Psychologv, 1974, 2, 151156.

Hoffman, H. S., \& Ison, J. R. Reflex modification in the domain of startle: I. Some empirical findings and their implications for how the nervous system processes sensory input. Psychological Review, 1980, 87, 175-189.

Hoffman, H. S., \& Searle, J. L. Acoustic variables in the modification of the startle reflex in the rat. Journal of Comparative and Physiological Psychologv, 1965, 60, 53-58.

Hoffman. H. S., \& Wible, B. L. Role of weak signals in acoustic startle. Journal of the Acoustical Society of America, 1970, 47, 489-497.

Ison, J. R.. \& Hammond, G. R. Modification of the startle reflex in the rat by changes in the auditory and visual environments. Journal of Comparative and Physiological Psvchologv. $1971.75 .435-452$ 
Ison, J. R., \& LeONARD, D. W. Effects of auditory stimuli on the amplitude of the nictitating membrane reflex of the rabbit (Oryctolagus cuniculus). Journal of Comparative and Physiological Psychology, 1971, 75, 157-164.

Ison, J. R., \& Silverste in, L. Acoustic startle reactions, activity, and background noise intensity, before and after lesions of medial cortex in the rat. Physiological Psychology, 1978, 6, 245-248.

Reiter, L. A., \& Ison, J. R. Reflex modulation and loudness recruitment. Journal of Auditory Research, 1980, in press.

Russo, J. M., Reiter, L. A., \& Ison, J. R. Repetitive exposure does not attenuate the sensory impact of the habituated stimulus. Journal of Comparative and Physiological Psychology, 1975, 88, 665-669.

SHEPHERD, G. M. The synaptic organization of the brain (2nd ed.). New York: Oxford University Press, 1979.
Steinberg, J. C., \& Gardner, M. B. The dependence of hearing impairment on sound intensity. Journal of the Acoustical Society of America, 1937, 9, 11-23.

Stitt, C. L., Hoffman, H. S., \& Marsh, R. R. Interaction versus independence of startle-modification processes in the rat. Journal of Experimental Psychology: Animal Behavior Processes, 1976, 2, 260-265.

Young, R. A., Cegavske, C. F., \& Thompson, R. F. Toneinduced changes in excitability of abducens motoneurons and the reflex path of nictitating membrane response in rabbit (Oryctolagus cuniculus). Journal of Comparative and Physiological Psychology, 1976, 90, 424-434.

(Received for publication January 25, 1980; revision accepted April 11, 1980.) 